\title{
Rectal operative bed: ?tumor? post- management changes ... positron emission tomography/computed tomography (PET/CT) can answer
}

\author{
Mohsen Ahmed Abdelmohsen ${ }^{1 *}$ (D, Walid Mohamed $\mathrm{Omar}^{2}$ and Ahmed Shaaban ${ }^{3}$
}

\begin{abstract}
Background: Rectal cancer is a common malignancy that continues to have a highly variable outcome. Local pelvic recurrence following surgery is usually leading to incurable disease. Surgical resection with stage-appropriate combinedmodality therapy is the mainstay in the treatment of rectal cancer. A difficulty has been reported in distinguishing the benign post-management findings from tumor recurrence. By adding the metabolic changes to the post-operative morphologic changes, combined positron emission tomography (PET) and computed tomography (CT) may offer clinically useful addition in operative bed evaluation.

Results: The study included 30 patients, 17 males (56.6\%) and 13 females (43.3\%). Suspicious operative bed findings included pre-sacral soft tissue density noted in 20 patients (66\%), anastomotic site mural thickening in five patients (16.6\%), and post-operative loco-regional nodes in five patients (16.6\%). Moreover, pre-sacral soft tissue focal avid uptake was noted in 17 patients (17/20, 85\%), and no uptake noted in three patients (3/20,15\%). Anastomotic site mural thickening was seen in five patients with positive uptake noted in three patients $(3 / 5,60 \%)$, and no uptake seen in two patients $(2 / 5,40 \%)$. Loco-regional nodes were seen in five patients with positive uptake in five patients. The most accurate SUV max cut-off value among studied cases was 3.5. Confirmation of PET/CT results was done by histopathological assessment in ten patients (33.3\%) and by follow up after 6 months in 20 patients (66.6\%) with recurrence noted in 15 patients (75\%) and normal post-operative findings noted in five patients (25\%).
\end{abstract}

Conclusion: PET/CT study is an effective tool for evaluation of suspicious colorectal oncologic operative bed findings noted in the routine $C T$ studies. PET/CT fusion images affect the clinical management by guiding further procedures (surgery and radiation therapy).

Keywords: Positron emission tomography, Computed tomography, Rectal cancer, Rectal operative bed, Pre-sacral tumefaction

\section{Background}

Rectal cancer is a common malignancy that have variable outcome; $30 \%$ of colorectal cancer are located in the rectum. Local invasion of the tumor into deeper tissues lead to peritoneal metastasis, whereas its spread through vascular structures and regional drainage lead to liver, lung, and

\footnotetext{
* Correspondence: Mohsenmohsenmd@yahoo.com;

mohsenmohsenmd@yahoo.com

${ }^{1}$ Present Address: Department of Radio-Diagnosis and Intervention, Faculty of Medicine, University of Alexandria, 10 Shamplion Street, Elazareeta, Alexandria, Egypt

Full list of author information is available at the end of the article
}

bone marrow metastasis. Rectal cancer can spread to adjacent structures such as adjacent adipose tissue, vagina, prostate, bladder, ureter, and the bony pelvis; there are different surgical approaches for management of operable cancer rectum, for example low anterior resection (LAR) procedure for recto-sigmoid operable malignancies with total mesorectal excision (TME), and abdomino-perineal resection (APR) for ano-rectal operable malignancies [1].

The post-operative field may show spectrum of normal post-operative findings, pre-sacral soft tissue density thickening notably following abdomino-perineal resection, 
anastomotic site mural thickening, locoregional subcentimeter lymph nodes, and pre-sacral fluid density collections [2, 3].

Unfortunately, recurrent neoplastic activities could be presented with morphologically similar changes especially pre-sacral soft tissue density and anastomotic site mural thickening $[4,5]$.

Conventional MDCT studies could not be totally conclusive to discriminate between the expected normal postoperative findings and early recurrent neoplastic process with difficult differentiation; moreover, the post-operative findings may persist for years or even indefinitely [5].

Histopathological assessment is the gold standard technique yet the lesions are not always accessible for biopsy; serial follow up is important in differentiation yet this is at the expense of missing the chance of early management and the patients may present with a progressive course or metastatic lesions in the follow up periods; post-operative MRI studies may be limited by the expected postoperative filed potential artifacts like surgical staples [6].

By adding the metabolic changes (expected increase glucose metabolism by the tumor tissues) to the postoperative morphologic changes, combined positron emission tomography (PET) and computed tomography (CT) may offer clinically useful addition in operative bed evaluation and early detection of recurrence by measuring the degree of accumulation of the fluorodeoxyglucose in the malignant cells helping to make differentiation [6-8].

\section{Methods}

This is a prospective analysis of 30 patients with pathologically proven rectal cancer managed by surgery with or without adjuvant radiotherapy with suspicious operative bed findings noted in CT colonography study done for all patients.

Suspicious CT data for malignancy then evaluated using PET/CT scanner, PET/CT was done at least 6 weeks after the surgical intervention or radiotherapy, using visual assessment method and semi-quantitative analysis measuring the standard uptake value (SUV) max and the normal would be the normal blood pool liver SUV max.

\section{Reference standard}

Ten patient lesions were subjected to a biopsy; serial follow up after 6 months was done in 20 patients as the standard references.

\section{Technique}

A hybrid PET/CT scanner (Siemens Biograph 64 PET/ CT scanner) was used in this study. Before the examination by $6 \mathrm{~h}$, the patients were instructed to fast, except for water. Avoidance of (extreme) exercise for at least $6 \mathrm{~h}$ before the study was asked to minimize FDG uptake in muscles and to reduce the false positive results. The
Table 1 Distribution of the studied patients according to different parameters

\begin{tabular}{lll}
\hline & $\begin{array}{l}\text { Number of the } \\
\text { patients ( } n: 30)\end{array}$ & Percentage \\
\hline Sex & 17 & $56.6 \%$ \\
$\quad$ Males & 13 & $43.3 \%$ \\
$\quad$ Females & & \\
Pre-operative tumor location & 11 & $36.6 \%$ \\
$\quad$ Upper rectal & 9 & $30 \%$ \\
Mid rectal & 10 & $33.3 \%$ \\
Low rectal & & \\
Confirmation of PET/CT findings & 10 & $33.33 \%$ \\
$\quad$ Histopathological assessment & 20 & $66.66 \%$ \\
Follow up after 6 months & &
\end{tabular}

allowable blood glucose level for the study was of $<150 \mathrm{mg} / \mathrm{dL}$. Voiding of urine was done before injection, and then approximately $5 \mathrm{MBq} / \mathrm{kg}$ body weight of FDG was injected. Further, 60-90 min later, the data were acquired [7]. The patients then were kept lying comfortably and were asked not to talk to avoid false positive uptake. A non-contrast-enhanced CT from midthigh to the skull was obtained $60 \mathrm{~min}$ after the tracer injection. Nuclear medicine consultant and radiology consultant interpreted the data at Siemens work station.

\section{Ethics approval and consent to participate}

All the patients included in this study gave written informed consent to publish the data contained within this study. Approval for this study was obtained from the Research Ethics Committee of our medical institute. All study procedures were carried out in accordance with the Declaration of Helsinki regarding research involving human subjects.

\section{Results}

The study included 30 patients, classified according the patients' sex into 17 males (56.6\%) and 13 females (43.3\%) (Table 1). Pre-operative tumor locations documented by pre-operative CT colonography studies were upper rectal in 11 patients (36.6\%), mid rectal in nine patients (30\%), and low rectal in ten patients (33.3\%). Suspicious operative bed findings in the follow-up imaging studies included pre-sacral soft tissue density is

Table 2 Distribution of the studied patients according to the suspicious rectal operative bed findings

\begin{tabular}{|c|c|c|}
\hline $\begin{array}{l}\text { Suspected operative } \\
\text { bed findings }\end{array}$ & Number of patients ( $N$ : 30) & Percentage \\
\hline Pre-sacral soft tissue density & 20 & $66 \%$ \\
\hline $\begin{array}{l}\text { Anastomotic site } \\
\text { mural thickening }\end{array}$ & 5 & $16.6 \%$ \\
\hline Loco-regional nodes & 5 & $16.6 \%$ \\
\hline
\end{tabular}



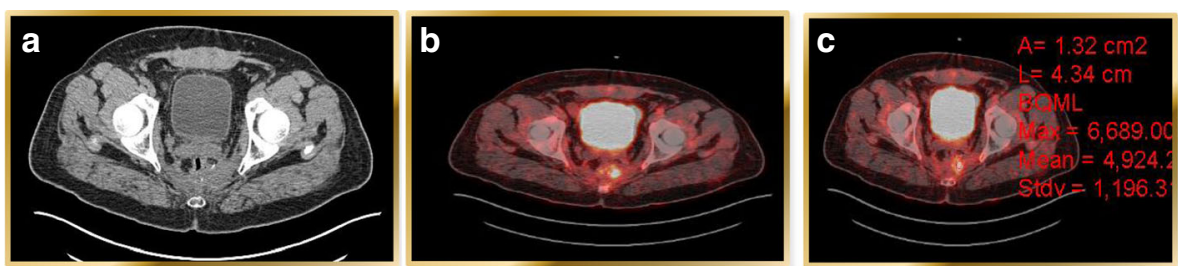

Fig. 1 a Post-operative CT study showing pre-sacral soft tissue density tumefaction. b PET/CT fused image with focal FDG uptake within the left para median aspect of the post-operative fibrosis using the visual assessment method. c PET/CT fused image with SUV max of the lesion 6.6 reflecting local recurrence confirmed by histopathological assessment

noted in 20 patients (66\%), anastomotic site mural thickening in five patients (16.6\%), and post-operative loco-regional nodes in five patients (16.6\%) (Table 2). Limited characterization based on CT findings with recommendations for serial follow up was documented.

PET/CT studies showed the following:

i. Patients with pre-sacral soft tissue density ( $n$ : 20$)$ :

A- Focal avid uptake noted in 17 patients $(17 / 20$, 85\%) (Fig. 1)

B- No uptake noted in three patients $(3 / 20,15 \%)$ (Fig. 2).

ii. Patients with anastomotic site mural thickening ( $n$ : 5):

A- Positive uptake noted in three patients $(3 / 5$, 60\%) (Fig. 3)

B- No uptake seen in two patients $(2 / 5,40 \%)$.

iii. Patients with loco-regional nodes in the operative bed $(n: 5)$ :

Five patients with positive uptake (Fig. 4) in the five patients (Table 3).

The SUV max cut-off value among studied cases was 3.5 , the highest SUV max value was 11, the lowest SUV max value was 1, and the median SUV max was 6. Confirmation of PET/CT results was done by either:

A- Histopathological assessment in ten patients (33.3\%), for accessible lesions for biopsy with positive results for recurrence.
B- Follow-up after 6 months in 20 patients (66.6\%) when no access for biopsy and follow up showed the following:

- Recurrence noted in 15 patients (n: 15/20) (75\%).

- Normal post-operative findings noted in five patients $(n: 5 / 20)$ (25\%).

Histopathological assessment was done by CT-guided percutaneous approach in the accessible lesions in ten patients with informative results; this constitutes another merit for PET/CT study for proper guiding for successful informative CT-guided biopsy with targeting the metabolically active areas (with positive FDG uptake) and avoiding the morphologically similar related scar/ granulation tissues reducing the false negative or noninformative results.

\section{Illustrative cases}

Case 1: 49-year-old male patient with history of cancer rectum managed by anterior resection on serial followup (Fig. 1).

Case2: 53-year-old female patient with history of mid third rectal cancer managed by anterior resection on serial follow up (Fig. 2).

Case 3: 50-year-old female patient with history of cancer rectum managed by staged rectal surgery with Hartman pouch creation (Fig. 3).

Case 4: 49-year-old male patient with history of mid third cancer rectum managed by anterior resection, presented in the 3 months follow up by left para rectal
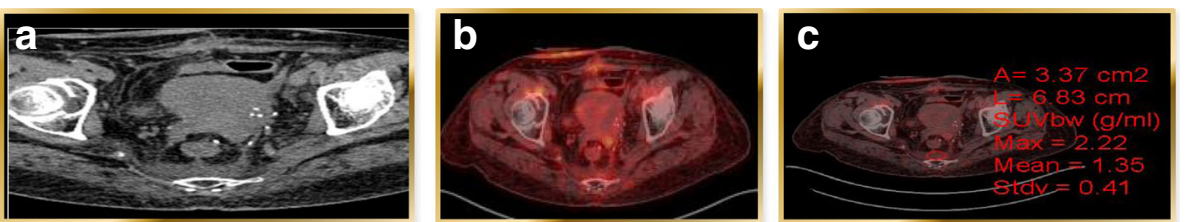

Fig. 2 a Post-operative CT study showing pre-sacral soft tissue density tumefaction. b PET/CT fused image with no appreciable FDG uptake within the post-operative fibrosis using the visual assessment method. c PET/CT fused image with SUV max of the lesion 2.2 with no suspicious areas of uptake 

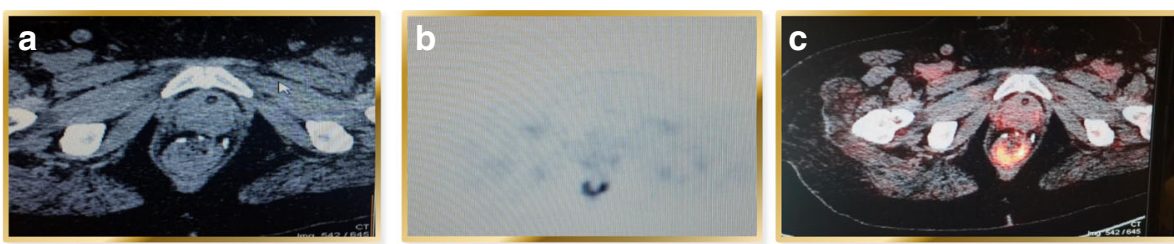

Fig. 3 a Post-operative CT study showing mild Hatman pouch anastomotic site mural thickening. b PET image with focal C shaped FDG uptake within the posterior aspect of the anastomotic site. c PET/CT fused image with avid uptake by the visual assessment method

soft tissue nodular lesion mostly enlarged discrete node (Fig. 4).

\section{Discussion}

The presence of numerous rectal cancer postoperative bed morphological changes offer a great challenge during serial follow-up of the rectal cancer post-operative patients; similar morphological patterns are noted in the normal post-operative fields as well as the evolving residual/recurrent neoplastic process.

Differentiation of post-therapeutic alterations attributable to scarring, inflammation, and necrosis from residual or recurrent tumors poses significant problems for CT [9].

Fibrotic or necrotic tissue that develops inside a tumor mass after treatment cannot be differentiated by $\mathrm{CT}$ from a viable malignant tumor, also tumor tissue within the post-operative fibrosis cannot be differentiated as well $[2,10]$. In this study, 17 patients had post-operative fibrosis at surgical bed noted at CT study without any suspicion of recurrence, but FDG $\mathrm{PET}$ and PET/CT detected recurrent tumoral tissue (Fig. 1).

In this study, focusing on the role of PET/CT in the post-operative rectal bed and ability to differentiate post-operative sequel from tumor recurrence, many studies focus upon rectal cancer metastatic potential; Van Custem et al. [11] focused upon the extra intestinal hepatic deposits diagnosis and management.
Paes et al. [12] also confirmed that the advantages of FDG PET/CT for the restaging are mostly attributed to the detection of FDG-avid, normal-sized lymph nodes.

In agreement-in this study-with Weston et al. [13], the accurate SUV max cutoff value was 3.5, yet there is discrepancy between the SUV max value in the literature, while Van Hoeij et al. [14] reported in their study the best cut off value of 11; Peng et al. [15] reported the best cut off value of 2.5 .

Gordin et al. [16] and Filippi et al. [17] stated that $\mathrm{PET} / \mathrm{CT}$ altered treatment management in more than $50 \%$ of the study population, while in this study PET/CT had changed patient management in 25 out of $30 \mathrm{pa}-$ tients $(83 \%)$ as we only included patients who were disease free after surgical removal of their primary tumor, referred to PET/CT due to a clinical or radiological suspicion of recurrence and this clarifies the discrepancy between our study and others in the impact of PET/CT on management.

The merits of the study are numerous; it can be helpful through metabolic and morphological characterization in determining risk-adapted treatment and long-term patient outcome by providing more accurate information about the rectal operative bed findings. Guidance for accurate biopsy can be helpful by revising the metabolic PET/CT data with reduction of the false negative or non-informative results.

Limitations of our study included the relative small number of studied patients, plus the ionizing radiation
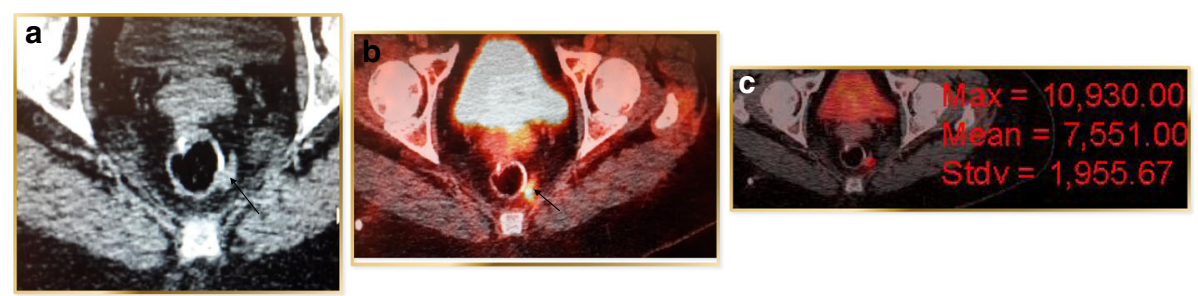

Fig. 4 a Post-operative CT study showing left para rectal soft tissue lesion (black arrow) mostly nodal. b PET/CT fused image with avid uptake of the lesion (black arrow) using the visual assessment method. c PET/CT fused image with SUV max of the lesion 10 reflecting high metabolic activity impressive of nodal deposits 
Table 3 Distribution of the studied patients according to the degree of FDG uptake by the suspicious rectal operative bed findings

\begin{tabular}{lll}
\hline Suspected operative bed findings & $\begin{array}{l}\text { Avid FDG } \\
\text { uptake }\end{array}$ & $\begin{array}{l}\text { Low FDG } \\
\text { uptake }\end{array}$ \\
\hline Pre-sacral soft tissue density $(n: 20)$ & 17 & 3 \\
Anastomotic site mural thickening $(n: 5)$ & 3 & 2 \\
Loco-regional nodes $(n: 5)$ & 5 & 0 \\
Total $(n: 30)$ & $25(83.3 \%)$ & $5(16.6 \%)$ \\
\hline
\end{tabular}

exposure risks in the PET/CT studies compared to relatively safer techniques like MRI examination that need further comparative studies assessment. Considerations about cost-effectiveness have not been part of this study but will be necessary, as they will play an increasing role in the near future as the clinical utility of PET/CT will lead to a change in the diagnostic and management strategy of cancer patients $[18,19]$.

\section{Conclusion}

Accurate re-staging of the post-operative rectal cancer patients can be reached by PET/CT assessment of the rectal operative bed as well as its metastatic potential with alteration of the patient management strategies.

\section{Abbreviations}

CT: Computerized tomography; PET: Positron emission tomography;

SUV: Standardized uptake value

\section{Acknowledgments}

Nil.

\section{Authors' contributions}

MAA reported the cases. WMO reported the cases and helped to draft the manuscript. AS helped to draft the manuscript. All authors read and approved the final manuscript.

\section{Funding}

This study had no funding from any resource.

\section{Availability of data and materials}

The data sets used and/or analyzed during the current study are available from the corresponding author on reasonable request.

\section{Ethics approval and consent to participate}

A written informed consent was obtained from all patients included in this study to publish this study data. Approval for this study was obtained from the Research Ethics Committee of Alexandria Faculty of Medicine. All study procedures were carried out in accordance with the Declaration of Helsinki regarding research involving human subjects.

\section{Consent for publication}

All the patients included in this study gave written informed consent to publish the data contained within this study.

\section{Competing interests}

The authors declare that they have no competing interests.

\section{Author details}

'Present Address: Department of Radio-Diagnosis and Intervention, Faculty of Medicine, University of Alexandria, 10 Shamplion Street, Elazareeta, Alexandria, Egypt. ${ }^{2}$ Department of Nuclear Medicine and Molecular Imaging,
National Cancer Institute, Cairo University, Cairo, Egypt. ${ }^{3}$ Department of Clinical and Experimental Surgery, Medical Research Institute Hospital, Alexandria University, Alexandria, Egypt.

Received: 1 August 2019 Accepted: 13 January 2020

Published online: 22 January 2020

\section{References}

1. Glynne-Jones R et al (2017) Rectal cancer: ESMO clinical practice guidelines for diagnosis, treatment and follow-up. Ann Oncol 28:22-40

2. Plodeck V, Rahbari NN, Weitz J, Radosa CG, Laniado M et al (2019) FDG-PET/ $\mathrm{MRI}$ in patients with pelvic recurrence of rectal cancer: first clinical experiences. Eur Radiol 29:422-428

3. São Julião GP, Habr-Gama A, Vailati BB, Araujo SEA, Fernandez LM, Perez RO (2017) New strategies in rectal cancer. Surg Clin North Am 97:587-604

4. Engstrom PF, Arnoletti JP, Benson AB 3rd, National Comprehensive Cancer Network et al (2009) NCCN clinical practice guidelines in oncology: colon cancer. J Natl Compr Canc Netw 7:778-831

5. Murcia Duréndez MJ, Frutos Esteban L, Luján J et al (2013) The value of ${ }^{18} \mathrm{~F}-\mathrm{FDG}$ PET/CT for assessing the response to neoadjuvant therapy in locally advanced rectal cancer. Eur J Nucl Med Mol Imaging 40:91-97

6. Lu YY, Chen JH, Chien CR et al (2013) Use of FDGPET or PET/CT to detect recurrent colorectal cancer in patients with elevated CEA: a systematic review and meta-analysis. Int J Color Dis 28:1039-1047

7. Zidan D, Hasan M, Tantawy M (2013) Postoperative restaging: PET/CT impact on diagnosis and management. Egypt J Radiol Nucl Med 44:321-329

8. Brush J, Boyd K, Chappell F et al (2011) The value of FDG positron emission tomography/computerised tomography (PET/CT) in pre-operative staging of colorectal cancer: a systematic review and economic evaluation. Health Technol Assess 15(35):1

9. Bailey JJ, Jordan EJ, Burke C, Ohliger MA, Wang ZJ et al (2018) Does extended PET acquisition in PET/MRI rectal cancer staging improve results? Am J Roentgenol 211:896-900

10. Sanli Y, Kuyumcu S, Ozkan ZG, Kilic L et al (2012) The utility of FDG-PET/CT as an effective tool for detecting recurrent colorectal cancer regardless of serum CEA levels. Ann Nucl Med 26:551-558

11. Van Cutsem E, Nordlinger B, Adam R et al (2006) Towards a pan-European consensus on the treatment of patients with colorectal liver metastases. Eur J Cancer 42:2212-2221

12. Paes F, Kalkanis D, Sideras P et al (2010) FDG PET/CT of extranodal involvement in non-Hodgkin lymphoma and Hodgkin disease. Radiographics 30:269-291

13. Weston BR, lyer RB, Qiao W et al (2010) Ability of integrated positron emission and computed tomography to detect significant colonic pathology: the experience of a tertiary cancer center. Cancer 116:1454-1461

14. Van Hoeij FB, Keijsers RG, Loffeld BC et al (2015) Incidental colonic focal FDG uptake on PET/CT :can the maximum standardized uptake value (SUV max ) guide us in the timing of colonoscopy ?.Eur. J Nucl Med Mol Imaging 42(1):66-71

15. Peng J, He $Y, X u J$ et al (2011) Detection of incidental colorectal tumors with ${ }^{18}$ F-labelled 2 -Flouro-2-deoxyglucose positron emission tomography /computed tomography scans: results of a prospective study. Colorectal Dis 13(11):374-378

16. Gordin A, Daitzchman M, Doweck I et al (2006) Fluorodeoxyglucosepositron emission tomography/computed tomography imaging in patients with carcinoma of the larynx: diagnostic accuracy and impact on clinical management. Laryngoscope 116:273-278

17. Filippi V, Malamitsi J, Vlachou F et al (2011) The impact of FDG-PET/CT on the management of breast cancer patients with elevated tumor markers and negative or equivocal conventional imaging modalities. Nucl Med Commun 32:85-90

18. SiegelR L, Miller KD, Jemal A (2017) Cancer statistics. CA Cancer J Clin 67:7-30

19. Han A, Xue J, Zhu D, Zheng J et al (2011) Clinical value of (18)F-FDG PET/CT in postoperative monitoring for patients with colorectal carcinoma. Cancer Epidemiol 35:497-500

\section{Publisher's Note}

Springer Nature remains neutral with regard to jurisdictional claims in published maps and institutional affiliations. 